Physics, Chemistry, and Dynamics of Interplanetary Dust

ASP Conference Series, Vol. 104, 1996

Bo A. S. Gustafson and Martha S. Hanner (eds.)

\title{
Dynamical Evolution of Interplanetary Dust due to Gravitational Scattering by Jupiter and the Inner Planets
}

\author{
Nikolai N. Gor'kavyi \\ Crimean Astrophysical Observatory, Simeiz 334242, Ukraine \\ Leonid M. Ozernoy ${ }^{1}$ and John C. Mather \\ Code 685, Goddard Space Flight Center, Greenbelt, MD 20771
}

\begin{abstract}
We introduce the continuity equation written in the coordinate space of the orbital elements (e.g. large semi-axis a vs. eccentricity $e$, etc.) to explore the density evolution of the zodiacal cloud. Emphasis is on those terms of the continuity equation which describe 'jumps' of particles in the $(a, e)$-space due to gravitational scattering of the interplanetary dust particles by planets, which represents one of the leading factors in the dynamical evolution of the zodiacal cloud. In the remainder (div-terms of the continuity equation), one can incorporate the known analytical expressions for the rates of gradual change of orbital elements due to the Poynting-Robertson and solar wind drags (Liou et al. 1995).
\end{abstract}

\section{Introduction}

The problem of dynamical evolution of interplanetary particles aims at finding the number density of particles, $n(\vec{r}, t)$, as a function of appropriate coordinates and time. As is known, four basic effects determine that evolution: (i) the Poynting-Robertson drag; (ii) resonance effects associated with Jupiter, Mars, Earth, and Venus; (iii) gravitational scattering of the particles by those planets; and (iv) mutual collisions of the particles (for reviews, see e.g. Dermott et al. 1993, 1994; Marzari \& Vanzani 1994). Effects (i) and (ii) result in a continuous motion of particles in the phase space, whereas effects (iii) and (iv) produce some 'jumps'. Indeed, for effects (i) and (ii) one can introduce a velocity at each point of the trajectory while effects (iii) and (iv) result in a practically instantaneous jump from a given point to another point.

\footnotetext{
${ }^{1}$ Computational Sciences Institute and Department of Physics \& Astronomy, George Mason
} University, Fairfax, VA 22030-4444 


\section{Basic Equation}

Both those continuous motions and jumps can be conveniently described by the continuity equation (Gor'kavyi, Ozernoy \& Mather 1996):

$$
\frac{\partial n\left(x_{i}, t\right)}{\partial t}+\frac{\partial}{\partial x_{i}}\left(n v_{i}\right)=N^{+}\left(x_{i}, t\right)-N^{-}\left(x_{i}, t\right),
$$

where $i=1 \ldots 6, v_{i}$ is velocity along the $i$-th axis in the phase space. This approach is very efficient since, instead of dealing with the motion of single particles, the continuity equation governs the density evolution of the entire cloud of dust particles in the phase space of the orbital elements. In the above equation, the div-term $\partial\left(n v_{i}\right) / \partial x_{i}$ describes 'slow' processes of change of particles' orbital elements such as the particle transport due to both the Poynting-Robertson drag and the resonance effects. The rates $v_{i}\left(\frac{\partial a}{\partial t}, \frac{\partial e}{\partial t}, \frac{\partial i}{\partial t \ldots}\right)$ for PR-drag (as well as for solar wind drag and the radiation pressure) have been determined by Liou, Zook, \& Jackson (1995). In the present paper, we make emphasis on the terms $N^{+}\left(x_{i}, t\right)$ and $N^{-}\left(x_{i}, t\right)$ in the r.h.s. of the equation, which are responsible for 'fast' processes of particles' gravitational scattering by the planets and the contact particle collisions (both mutual and with the planets). $N^{+}\left(x_{i}, t\right)$ accounts for the 'birth' of particles in the given point $x_{i}$ due to the jumps from the other points, and $N^{-}\left(x_{i}, t\right)$ accounts for the 'death' of particles due to their escape from $x_{i}$.

For direct scattering with the planets due to close encounters $N^{+}(a, e)=$ $\int W\left(a, e, a^{\prime}, e^{\prime}\right) n\left(a^{\prime}, e^{\prime}\right) d a^{\prime} d e^{\prime}$, where $W\left(a, e, a^{\prime}, e^{\prime}\right)$ is the probability of particle transfer from the point $\left(a^{\prime}, e^{\prime}\right)$ to the point $(a, e)$ by a jump. The value of $N^{-}(a, e)$ is given by $N^{-}(a, e)=n(a, e) P(a, e) \equiv n(a, e) \int W\left(a, e, a^{\prime}, e^{\prime}\right) d a^{\prime} d e^{\prime}$ where $P(a, e)$ is the integral probability of the particle escaping from the point $(a, e)$ to the points $\left(a^{\prime}, e^{\prime}\right)$ to which the gravitational scattering occurs. Gravitational scattering of a dust particle by a planet, being an elastic process, keeps the total energy of the particle constant (as measured in the coordinate system moving with the planet). As a result, the dynamical trajectory of the particle in the $(a, e)$-space is described by the Tisserand criterion (e.g. Roy 1978): $\left(a_{P} / 2 a\right)+\sqrt{\left(a / a_{P}\right)\left(1-e^{2}\right)} \cos i=C$. In a fast process such as gravitational scattering, $C$ can be taken as a constant (as well as the particle's energy). The use of the Tisserand criterion allows to reduce the number of computercalculated orbital elements of particles by 1 .

\section{Numerical Results}

For convenience of computations, let us replace $W$ as a continuous function by a matrix.

We have created a set of computer codes for fast computations of the elements of the scattering matrix $W$ (Gor'kavyi, Ozernoy \& Mather 1996). These codes determine the probability of the jump of particles from a given to another point in the $(a, e)$-space. Formally, the total number of elements in the matrix $W$ is very large. However, in practice there is no need to compute all the matrix elements in advance: it is necessary to compute those (and only those) matrix 


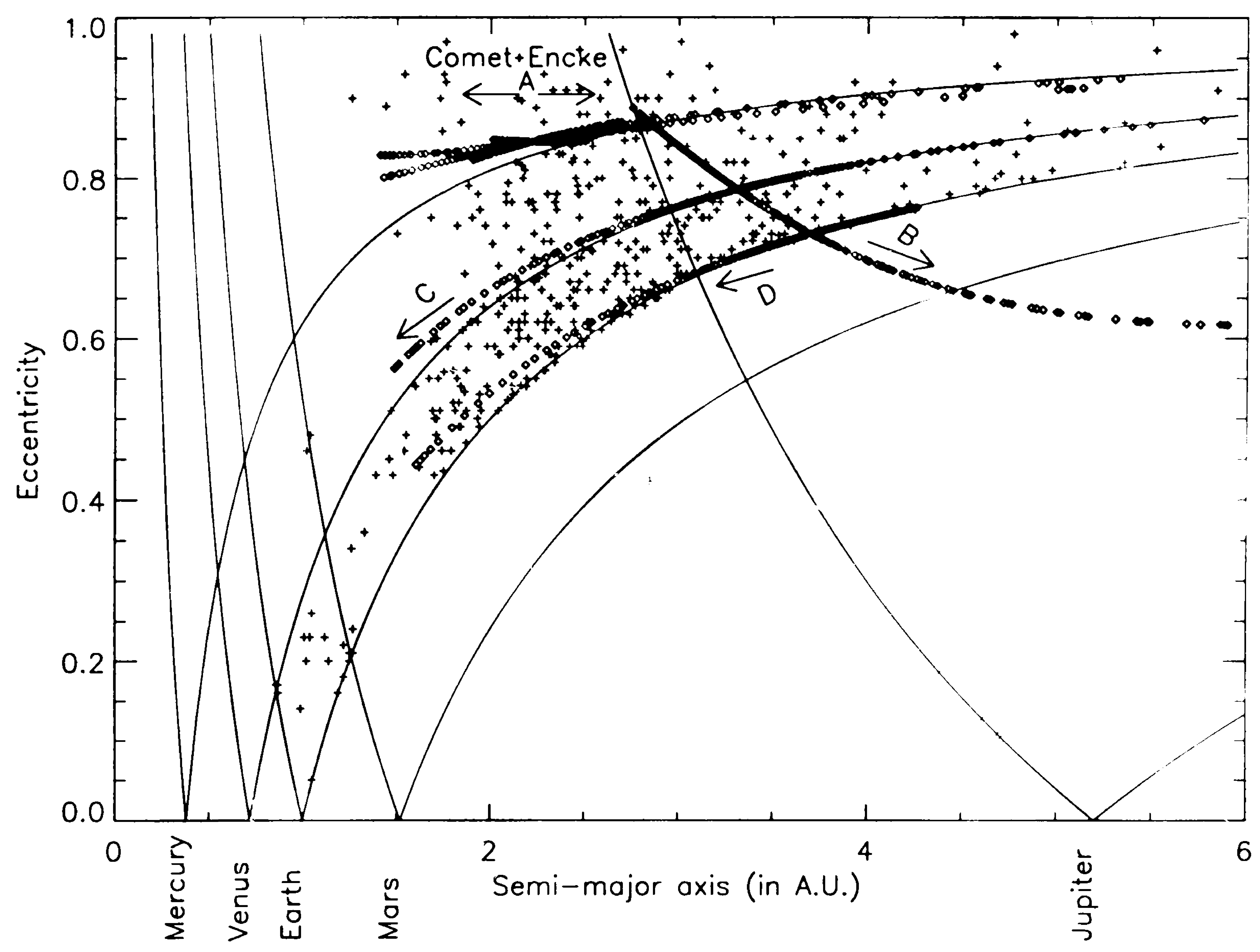

Figure 1. Three stages of the scattering of comet Encke particles with initial $a_{0}=2.22, e_{0}=0.846$ in the $(a, e)$-space. Computer-calculated particle orbits are represented by open diamonds. First stage (labeled as A): scattering of comet Encke particles due to perturbations from all four inner planets Second stage (labeled as B): scattering of particles by Jupiter. Third stage (labeled as C): the next scattering path of the comet Encke particles due to perturbations from Venus. D labels the next scattering path of the particles due to gravitational scattering by the Earth. Crosses show meteor orbits from photographic, radar, and visual observations (Terentjeva 1966).

elements which contain the positions of the particles after the scattering. Therefore, the result of computations of the particle evolutionary tracks determines the elements of the matrix $W$. In other words, this matrix turns out to be selffilling. The matrix $W$ is written in the form of a computer file that contains the complete information about the fate of a particle.

To illustrate how our method works, we consider the evolution of particles whose initial positions in the $(a, e)$-space are given by: $a_{0}=2.22, e_{0}=0.846$, which corresponds to the inflow of particles from the Encke comet. As our purposes are just illustrative, it is reasonable to simplify the problem as much as possible: the orbits of all the planets are taken to be circular, the problem is 2$\mathrm{D}(i=0$ both for the particles and planets), the effect of solar radiation pressure and the resonance effects are neglected, and an accuracy of determination of the particles' coordinates in the $(a, e)$-space is taken to be $\delta a, \delta e=0.01$.

Figure 1, which shows several stages of scattering of particles from comet 
Encke $\left(a=2.2, e_{0}=0.846\right)$ in the $(a, e)$-space, illustrates one of the most effective ways of the dynamical evolution of cometary particles due to gravitational scattering by Jupiter and the inner planets. It is of interest to compare these results with observations indicating the presence of numerous meteor streams (Cook 1973), fireballs (Terentjeva 1990), and single meteors (Terentjeva 1966); meteor orbits are plotted on Fig. 1. Solar pressure for those particles, owing to their large sizes, is negligible.

\section{Conclusions}

The main results of the present analysis are the following:

- The continuity equation is a convenient tool to describe both the continuous motion of particles in the phase space (due to the Poynting-Robertson drag and the resonance effects) and jumps (due to particle collisions and gravitational scattering of particles by the planets.

- While considering the gravitational scattering alone, the evolutionary tracks of dust particles in the $(a, e)$-space are located very close to the Tisserand criterion curves. Our numerical computations yield the rate of evolution of particle density along the Tisserand curves.

Our codes compute the elements of the scattering matrix $W$ that describes the transport of interplanetary particles in the $(a, e)$-space. A success in calculating of both the matrix $W$ and the terms $N^{+}\left(x_{i}, t\right)$ and $N^{-}\left(x_{i}, t\right)$ in the continuity equation make possible further solution of the continuity equation for density of the zodiacal particles in more sophisticated conditions, with incorporation of the div-term $\partial\left(n v_{i}\right) / \partial x_{i}$ that describes the particle transport due to the Poynting-Robertson drag, solar wind, etc.

\section{References}

Cook, A.F 1973, in Evolutionary and Physical Properties of Meteoroids, Proc. IAU Coll. No. 13, (eds. C.L.Hemenway et al.) Washington, 183

Dermott, S.F., Durda, D.D., Gustafson, B.A..S., Jayaraman, S., Liou, J.C., \& Xu, Y.L. 1993, in Asteroids, Comets, Meteors 1993 (eds. A. Milani et al.), 127

Dermott, S.F., Jayaraman, S., Xu, Y.L., Gustafson, B.A.S., \& Liou, J.C. 1994, Nature 369,719

Gor'kavyi, N.N., Ozernoy, L.M., \& Mather, J.C. 1996, ApJ (submitted)

Liou, J.-C., Zook, H.A., Jackson, A.A. 1995, Icarus 116, 186

Marzari, F。 \& Vanzani, V 1994, A\&A 283, 275

Roy, A.E. 1978, Orbital Motion. Bristol, A. Hilger Ltd.

Terentjeva, A.K. 1966, "Malye meteornye roi", in "Issledovanie meteorov", N.1, Moscow, Nauka. 1966. 62

Terentjeva, A.K. 1990, in Asteroids, Comets, Meteors. III (eds. C.-I. Lagerquist, H. Rickman, B.A. Lindblad, \& M. Lingren). Uppsala, 579 